\title{
Correlation between cortisol levels and concurrent infection for hepatitis B cirrhosis patients and countermeasure analysis
}

\author{
JIAN ZHANG, JUNWEI MA, HONGXIN WANG and JUNHONG LI \\ Department of Emergency, Beijing YouAn Hospital, Capital Medical University, Beijing 100069, P.R. China
}

Received August 8,2017; Accepted December 13, 2017

DOI: 10.3892/etm.2018.5738

\begin{abstract}
The study assessed the correlation between cortisol (COR) levels and concurrent infection for the patients with hepatitis B cirrhosis for corresponding countermeasure analysis. In total, 86 patients with hepatitis B cirrhosis (non-infection group) and 32 patients with hepatitis B cirrhosis complicated with infection (infection group) who were diagnosed and treated in the Beijing YouAn Hospital from March 2014 to March 2017 were selected. The fasting venous blood of all the patients was drawn to detect COR, cortisol binding globulin $(\mathrm{CBG})$, blood routine indexes, C-reactive protein (CRP), procalcitonin (PCT), endotoxin and other indicators. The relative expression of CBG mRNA was detected by reverse transcription quantitative polymerase chain reaction (RT-qPCR). The differences and correlation of COR levels between the infection and non-infection groups were compared and analyzed. The concentrations of COR and CBG were decreased with the increase of Child-Pugh grade, and the difference was statistically significant $(\mathrm{P}<0.05)$. COR, CBG and free cortisol (FC) concentrations with the same Child-Pugh grade in the non-infection group were higher than those in the infection group $(\mathrm{P}<0.05)$. COR, CBG and FC concentrations of abdominal infection complicated with sepsis or abdominal infection complicated with pulmonary infection were lower than those of simple abdominal infection $(\mathrm{P}<0.05)$. The relative expression of $\mathrm{CBG}$ mRNA was detected by RT-qPCR, which also showed that: for Child-Pugh grade, grade $\mathrm{A}>$ grade $\mathrm{B}>$ grade $\mathrm{C}(\mathrm{P}<0.05)$, non-infection group $>$ infection group $(\mathrm{P}<0.05)$, abdominal infection + sepsis group and abdominal infection + pulmonary infection group were lower than the simple abdominal infection group $(\mathrm{P}<0.05)$. The values of white blood cells (WBC), neutrophils, CRP, PCT and endotoxin in the infection group were higher than those in
\end{abstract}

Correspondence to: Dr Junhong Li, Department of Emergency, Beijing YouAn Hospital, Capital Medical University, 8 Youanmen Wai Street, Beijing 100069, P.R. China

E-mail: junhong_li123@163.com

Key words: hepatitis B cirrhosis, cortisol, concurrent infection, correlation, cortisol binding globulin the non-infection group, and the differences were statistically significant $(\mathrm{P}<0.05)$. COR, CGB and $\mathrm{FC}$ were negatively correlated with inflammatory indexes such as WBC, neutrophils, CRP, PCT and endotoxin. The $r$ value of COR and FC in the non-infection group was 0.678 , while that of OR and FC in the infection group was 0.787 . COR was positively correlated with FC before and after infection. The results of the study show that the cortisol levels of patients with hepatitis B cirrhosis are significantly correlated with whether infected or not, levels of disease condition and infection types, and can be used as sensitive indicators of hepatitis B cirrhosis infection.

\section{Introduction}

Liver cirrhosis has clinical features such as high incidence, difficult curability and high fatality rate (1). Hepatitis B virus-related cirrhosis is the main type of liver cirrhosis. With the development of hepatitis B disease, liver cirrhosis occurs. According to statistics, for the patients with hepatitis B cirrhosis, the 5-year survival rate is approximately $50 \%$, while the survival rate is lower than $20 \%$ in the decompensated period, leading to severe impact on the patients' quality of life of the elderly in China $(2,3)$.

The concurrent infection caused by bacteria and other pathogens is one of the important factors resulting in the difficulties to successfully treat patients with hepatitis B cirrhosis, and can lead to death (4). In recent years, it has been reported that most patients with hepatitis B cirrhosis complicated with infection are accompanied with a decrease of cortiadrenal function, and thereby liver function is indirectly affected, which makes the disease difficult to treat (5-7).

In this study, the cortisol secreted by the adrenal cortex was the focal point, the changes of the infected and uninfected cortisol and serum inflammatory factors of patients with hepatitis B cirrhosis were compared, and the correlation between cortisol (COR) and concurrent infection as well as the countermeasures were analyzed.

\section{Materials and methods}

Materials. According to the criteria of the Guideline of Prevention and Treatment for Chronic hepatitis B (2010 version), 86 patients who were diagnosed and treated in the Beijing YouAn Hospital from March 2014 to March 2017 were selected as the non-infection group. This group comprised 
58 males and 28 females, aged 25-72 years, with an average age of $54.32 \pm 15.41$ years. According to the Child-Pugh grade, there were 7 cases with grade A, 38 cases with grade B and 41 cases with grade $\mathrm{C}$. Thirty-two cases of patients were included in the infection group, including 21 males and 11 females aged 24-73 years, with an average age of $55.14 \pm 16.03$ years. There were 2 cases with grade A, 14 cases with grade B and 16 cases with grade $\mathrm{C}$. Of the 32 cases, 14 cases had simple abdominal infection, 10 cases had abdominal infection complicated with sepsis, and 8 cases had abdominal infection complicated with pulmonary infection.

Exclusion criteria were: i) Patients with other virus infections except HBV; ii) patients with non-alcoholic steatohepatitis, tumor, alcoholic liver disease, cardio-cerebrovascular disease and other major diseases; iii) patients with abnormal adrenal cortex function and any patients who had taken drugs affecting the secretion of cortisol within the last six months.

The differences were not statistically significant regarding age, sex, weight, disease grading and other indexes between the two groups of patients ( $P>0.05)$, and thus comparable.

This study was approved by the Hospital Ethics Committee of the Beijing YouAn Hospital (Beijing, China), and made an introduction to the selected patients to ensure that informed consent was signed by them and that they were fully informed.

Reagents. COR chemiluminescence kit (Beckman Coulter, Brea, CA, USA), cortisol binding globulin (CBG) enzyme-linked immunosorbent assay (ELISA) kit (Shanghai Jiang Lai Biotechnology Co., Ltd., Shanghai, China), TRIzol (Thermo Fisher Scientific, Waltham, MA, USA), chloroform, isopropanol (Beijing Chemical Factory, Beijing, China), moloney murine leukemia virus (M-MLV) reverse transcriptase, DNase I (both from Thermo Fisher Scientific), SYBR $^{\circledR}$ Premix Ex Taq ${ }^{\text {TM }}$ II (Takara, Dalian, China), and synthetic primers (BGI Genomics, Shenzhen, Guangdong, China) were used in the present study.

Blood routine test. Fasting venous blood $(15 \mathrm{ml})$ was collected from all the patients in the morning, and $7 \mathrm{ml}$ was used in the tests for related inflammatory indexes using a Roche C501 automatic biochemical analyzer (Roche Diagnostics, Basel, Switzerland) including white blood cells (WBC), neutrophils, procalcitonin (PCT), endotoxin and C-reactive protein (CRP).

Detection of cortisol concentration. The collected $7 \mathrm{ml}$ fasting venous blood was centrifuged at $1,300 \mathrm{~g} / \mathrm{min}$ for $15 \mathrm{~min}$ at $4^{\circ} \mathrm{C}$ in order to collect serum. The concentrations of COR and CBG in the serum were measured by corresponding kits. The concentration of free cortisol (FC) was calculated using the Coolens formula: $\mathrm{FC}$ concentration $\left.(\mathrm{mmol} / \mathrm{l})=\mathrm{Z}^{2}+0.122 \mathrm{c}\right) 1 / 2-Z$; and the coefficient $\mathrm{Z}$ was calculated, $\mathrm{Z}=0.0167+0.182$ (T-C), while $\mathrm{T}$ was the concentration of $\mathrm{COR}$, and $\mathrm{C}$ was the concentration of CBG (8).

mRNA expression detection of $C B G$ gene. RNA was extracted from $1 \mathrm{ml}$ whole blood by TRIzol. The procedure was strictly performed as per the protocol following the instructions, and the concentration was measured. RNA $(1 \mu \mathrm{g})$ was taken to be used for reverse transcription reaction by reverse transcriptase kit to obtain cDNA. The concentration
Table I. RT-PCR primer sequences of CBG and $\beta$-actin mRNA.

\begin{tabular}{lc}
\hline Gene names & \multicolumn{1}{c}{ Primer sequences } \\
\hline$C B G$ & 5'-TAGCCCAGCCATCCTC-3' \\
& 3'-GTGCTCGACTGCAACATC-5' \\
$\beta$-actin & 5'-TCAGGTCATCACTATCGGCAAT-3' \\
& 3'-AAAGAAAGGGTGTAAAACGCA-5'
\end{tabular}

CBG, cortisol binding globulin.

of cDNA was adjusted, and the levels of mRNA and relative expression of different groups were measured using Bio-Rad CFX 96 PCR (Hercules, CA, USA) instrument in accordance with the instructions of the SYBR ${ }^{\circledR}$ Premix Ex Taq $^{\mathrm{TM}}$ II kit. The corresponding primer sequences are shown in Table I (9).

Statistical analysis. Statistical Product and Service Solutions (SPSS) 19.0 (Hangzhou NewGrand Software Co., Ltd., Hangzhou, China) was used for data processing. Measurement data are shown as mean $\pm \mathrm{SD}$, and were detected by student's t-test. The Kruskal-Wallis test was used for the enumeration data and multiple comparisons (Tables I and II). Analysis of variance was performed, and then pairwise comparison. Pearson's test was used for the correlation analysis between factors. $\mathrm{P}<0.05$ was considered statistically significant (10).

\section{Results}

Comparisons of cortisol levels for the three Child-Pugh grades in the two groups. The concentrations of COR and CBG decreased with the increase of Child-Pugh grades in the infection and non-infection groups, and the differences were statistically significant $(\mathrm{P}<0.05)$. The concentrations of $\mathrm{COR}$, $\mathrm{CBG}$ and $\mathrm{FC}$ at the same grade in the non-infection group were higher than those in the infection group $(\mathrm{P}<0.05)$ (Table II). The relative expression of CBG was detected by RT-qPCR, which also showed that grade $\mathrm{A}>$ grade $\mathrm{B}>$ grade $\mathrm{C}$ $(\mathrm{P}<0.05)$, and non-infection group $>$ infection group $(\mathrm{P}<0.05)$ (Fig. 1).

Comparisons of cortisol levels among different infection types in the infection group. The concentrations of COR, CBG and FC for abdominal infections complicated with sepsis or pulmonary infection were lower than those for simple abdominal infection (Table III). The relative expression of CBG was detected by RT-qPCR, which showed that the group of abdominal infection complicated with sepsis and the group of abdominal infection complicated with pulmonary infection were lower than the group of simple abdominal infection $(\mathrm{P}<0.05)$ (Fig. 2).

Comparisons of related infection indexes between the two groups. The values of WBC, neutrophils, CRP, PCT and endotoxin in the infection group were higher than those in the non-infection group, and the differences were statistically significant $(\mathrm{P}<0.05)$ (Table IV). 
A

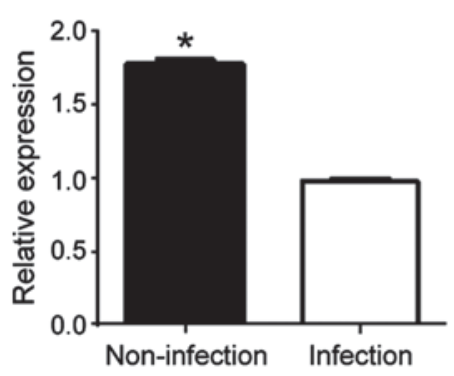

B

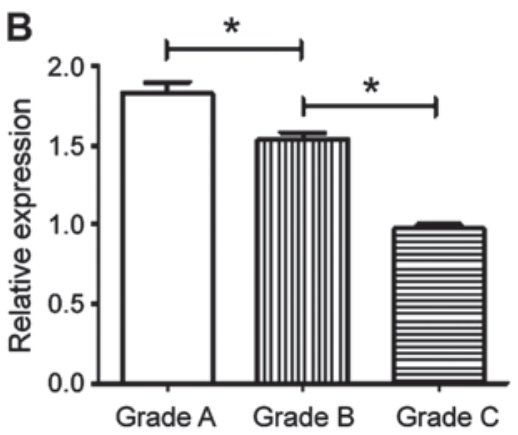

Figure 1. The relative expression of cortisol binding globulin (CBG) is detected by reverse transcription-quantitative polymerase chain reaction (RT-qPCR). (A) The non-infection group is higher than the infection group in (" $\mathrm{P}<0.05)$. (B) Grade $\mathrm{A}>$ grade $\mathrm{B}>$ grade $\mathrm{C}(\mathrm{P}<0.05)$.

Table II. Comparisons of cortisol levels for the three Child-Pugh grades in the two groups, mean \pm SD.

\begin{tabular}{llcccccc}
\hline & \multicolumn{3}{c}{ Non-infection group } & & \multicolumn{3}{c}{ Infection group } \\
\cline { 2 - 3 } Observation indexes & Grade A & Grade B & Grade C & & Grade A & Grade B & Grade C \\
\hline COR $(\mu \mathrm{g} / \mathrm{dl})$ & $14.98 \pm 4.34$ & $13.87 \pm 4.78^{\mathrm{a}}$ & $11.98 \pm 4.07^{\mathrm{a}, \mathrm{b}}$ & & $13.76 \pm 5.13^{\mathrm{c}}$ & $12.12 \pm 4.97^{\mathrm{a}, \mathrm{c}}$ & $10.43 \pm 3.76^{\mathrm{a}-\mathrm{c}}$ \\
CGB $(\mu \mathrm{g} / \mathrm{ml})$ & $47.43 \pm 11.21$ & $36.54 \pm 9.87^{\mathrm{a}}$ & $28.42 \pm 9.06^{\mathrm{a}, \mathrm{b}}$ & & $38.21 \pm 14.32^{\mathrm{c}}$ & $29.56 \pm 12.01^{\mathrm{a}, \mathrm{c}}$ & $22.36 \pm 9.65^{\mathrm{a}-\mathrm{c}}$ \\
FC $(\mathrm{nmol} / \mathrm{l})$ & $95.21 \pm 3.21$ & $94.72 \pm 4.54^{\mathrm{a}}$ & $94.77 \pm 3.12^{\mathrm{a}}$ & & $89.21 \pm 4.21^{\mathrm{c}}$ & $88.67 \pm 3.87^{\mathrm{a}, \mathrm{c}}$ & $87.32 \pm 2.63^{\mathrm{a}, \mathrm{c}}$ \\
\hline
\end{tabular}

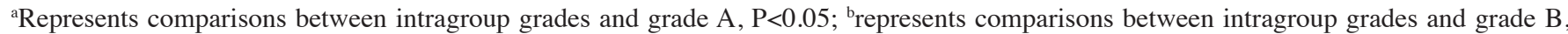
$\mathrm{P}<0.05$; 'represents comparisons among groups at the same grade, $\mathrm{P}<0.05$. CBG, cortisol binding globulin; COR, cortisol; FC, free cortisol.

Table III. Comparisons of cortisol levels among different infection types in the infection group (mean \pm SD).

\begin{tabular}{lcccc}
\hline Infection types & No. $(\mathrm{A} / \mathrm{B} / \mathrm{C})$ & $\mathrm{COR}(\mu \mathrm{g} / \mathrm{dl})$ & CGB $(\mu \mathrm{g} / \mathrm{ml})$ & FC $(\mathrm{nmol} / \mathrm{l})$ \\
\hline Abdominal infection & $14(2 / 6 / 6)$ & $13.81 \pm 4.54$ & $40.32 \pm 13.12$ & $91.43 \pm 4.08$ \\
Abdominal + sepsis & $10(0 / 5 / 5)$ & $10.97 \pm 3.54^{\mathrm{a}}$ & $23.32 \pm 9.87^{\mathrm{a}}$ & $86.67 \pm 3.65^{\mathrm{a}}$ \\
Abdominal + pulmonary & $8(0 / 3 / 5)$ & $10.43 \pm 3.54^{\mathrm{a}}$ & $22.98 \pm 11.08^{\mathrm{a}}$ & $86.18 \pm 3.76^{\mathrm{a}}$
\end{tabular}

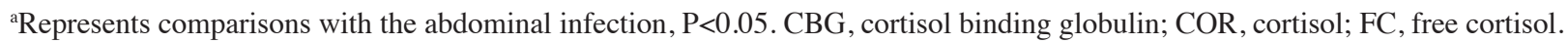

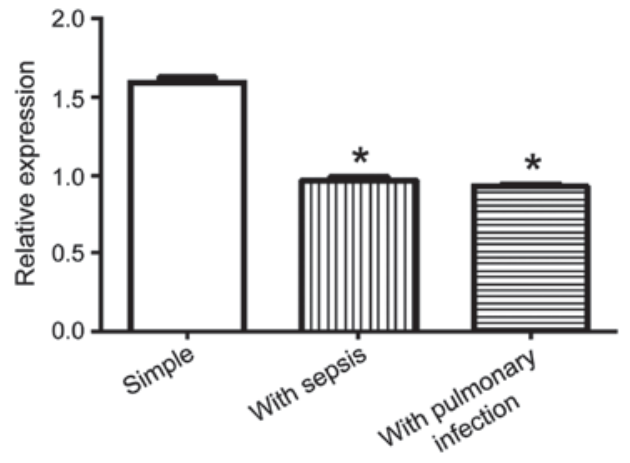

Figure 2. Relative mRNA expression of 3 groups of cortisol binding globulin $(C B G)$ genes. The group of abdominal infection complicated with sepsis and the group of abdominal infection complicated with pulmonary infection are lower than the group of simple abdominal infection $(" \mathrm{P}<0.05)$.

Correlation analysis of cortisol and related infection indexes. COR, CGB and FC were negatively correlated with such indexes as WBC, neutrophils, CRP, PCT and endotoxin. The specific Pearson's coefficients are shown in Table V.

Correlation analysis of COR and FC. The r value of COR and FC in the non-infection group was 0.678 , while that of of COR and FC in the infection group was 0.787 ; infection group was positively correlated with non-infection group, and COR was positively correlated with FC (Fig. 3).

\section{Discussion}

Hepatitis induced by HBV is one of the most common forms of hepatitis induced by various viruses. Liver cirrhosis caused by this is one of the major diseases in the world. Liver cirrhosis is commonly complicated with infection, liver ascites and liver cancer (11). Infection is the common complication in liver cirrhosis. Liver cirrhosis complicated with infection often has long recovery time and poor prognostic result, and the mortality rate is $60 \%$ (12). Cortisol, also known as hydrocortisone and 
Table IV. Comparisons of related infection indexes between the two groups (mean $\pm \mathrm{SD})$.

\begin{tabular}{|c|c|c|c|c|}
\hline Infection indexes & Non-infection group & Infection group & t-values & P-value \\
\hline WBC (x109/1) & $5.58 \pm 2.89$ & $8.49 \pm 4.99$ & 3.345 & 0.002 \\
\hline Neutrophils (\%) & $67.87 \pm 6.76$ & $77.87 \pm 9.98$ & 5.012 & 0 \\
\hline $\mathrm{CRP}(\mu \mathrm{g} / \mathrm{l})$ & $14.61 \pm 3.76$ & $20.51 \pm 5.32$ & 3.145 & 0.036 \\
\hline PCT (ng/ml) & $1.29 \pm 0.64$ & $1.79 \pm 0.82$ & 3.169 & 0.041 \\
\hline Endotoxin (pg/ml) & $60.76 \pm 29.87$ & $271.08 \pm 138.76$ & 3.132 & 0.024 \\
\hline
\end{tabular}

CBG, cortisol binding globulin; COR, cortisol; FC, free cortisol; PCT, procalcitonin.

Table V. Correlation analysis of cortisol and related infection indexes.

\begin{tabular}{lccccc}
\hline Indexes & WBC $\left(x 10^{9} / \mathrm{l}\right)$ & Neutrophils, sec $(\%)$ & CRP $(\mu \mathrm{g} / \mathrm{l})$ & PCT (ng/ml) & Endotoxin $(\mathrm{pg} / \mathrm{ml})$ \\
\hline COR $(\mu \mathrm{g} / \mathrm{dl})$ & -0.657 & -0.598 & -0.712 & -0.698 & -0.812 \\
CGB $(\mu \mathrm{g} / \mathrm{ml})$ & -0.712 & -0.608 & -0.743 & -0.765 & -0.784 \\
FC $(\mathrm{nmol} / \mathrm{l})$ & -0.687 & -0.654 & -0.733 & -0.654 & -0.765 \\
\hline
\end{tabular}
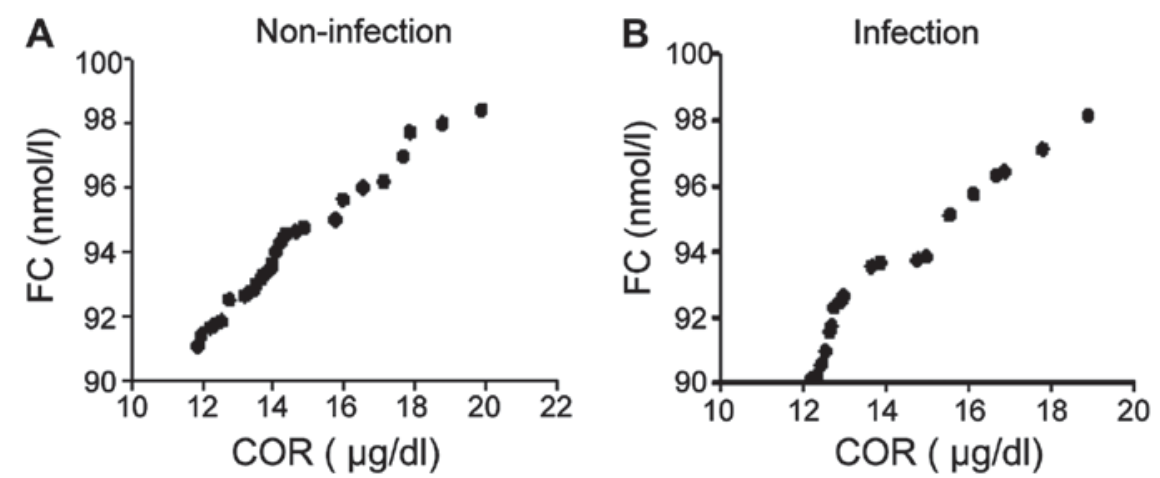

Figure 3. Correlation analysis of cortisol (COR) and free cortisol (FC) before and after infection. (A) COR is positively correlated with FC in the non-infection group $(r=0.687)$. (B) COR is positively correlated with FC after infection $(r=0.787)$.

which is produced by the adrenal gland, mainly exists in the form of binding state and free state. Cortisol has a particularly important effect in controlling mood and health, immune cells and inflammation, connection between blood vessel and blood pressure, as well as maintaining bones, muscles, skin and other organs. Cortisol generally maintains the stability of blood pressure and controls excessive inflammation in the case of pressure state $(13,14)$.

Cirrhosis can be divided into grade A, B and C according to Child-Pugh score from low to high in accordance with its development degree. Especially in later period, the secretion of COR often becomes abnormal, which is largely related to the synthesis and expression of CBG being affected $(15,16)$. In this study, the results showed that the concentrations of COR and CBG decreased with the increase of Child-Pugh grade in either the infection group or non-infection group, and the differences were statistically significant $(\mathrm{P}<0.05)$. The relative expression of CBG was detected by RT-qPCR, which showed that: grade $\mathrm{A}>$ grade $\mathrm{B}>$ grade $\mathrm{C}(\mathrm{P}<0.05)$. Previous findings have shown that $50-70 \%$ of the patients suffering from shock due to cirrhosis combined with infection have adrenal insufficiency, and the damaged adrenal cortical function is bound to affect the level of cortisol in vivo (17). In this study, the results also verified that the concentrations of COR, CBG and FC in the infection group were lower than those in the non-infection group whether on the whole or at the same Child-Pugh grade, and RT-qPCR also confirmed that the expression of $C B G$ gene in the infection group was lower than that in the non-infection group, which was consistent with the findings of Hamrahian et al, who identified that, the expression of $C B G$ gene declined rapidly with the deepening of infection extent (18).

This study also showed that the concentrations of COR, CBG and FC for abdominal infection complicated with sepsis or pulmonary infection were lower than those for simple abdominal infection $(\mathrm{P}<0.05)$. The relative expression of CBG was detected by RT-qPCR showing that the group of abdominal infection complicated with sepsis or pulmonary infection were lower than the simple abdominal infection $(\mathrm{P}<0.05)$. The results suggests that different infection sites and types can also lead to the decrease of the cortisol level in vivo, especially for more than two concurrent infections, which may 
be greatly associated with that of more serious infection. Thus, the metastasis of abdominal infection to infect other organs exacerbates the disease.

WBC, neutrophil percentage, CRP, PCT and endotoxin are the most common and sensitive indexes used to measure infection and the most intuitive indexes for infection extent of blood routine at present, and their elevated degree is directly proportional to disease degree of inflammation (19). The present study also verified that the above indexes of the hepatitis B cirrhosis patients complicated with infection were higher than those of the hepatitis B cirrhosis patients without infection. In addition COR, CGB and FC were negatively correlated with such indexes as WBC, neutrophils, CRP, PCT and endotoxin by further study for the correlation between infection indexes and cortisol. Results show that the cortisol levels of hepatitis B cirrhosis patients are significantly correlated whether infected or not, and grades of condition and infection types can be used as sensitive indexes to control hepatitis B cirrhosis infection.

In conclusion, the content of cortisol in vivo can be used as an important index to treat and prevent infection. Therefore, to reduce the probability of infection, grading of hepatitis B cirrhosis should be predicted by the detection of cortisol concentrations. Medical treatment should be taken as early as possible according to different disease grades, and nursing measures should be reinforced. Adrenocortical function should be improved, and the cortisol level should be kept at a reasonable range, in order to indirectly reduce the occurrence of infection. In addition, nursing care for cirrhosis patients should be enhanced, in order to reduce the occurrence rates of psychological fear and unforeseen circumstances.

\section{Competing interests}

The authors declare that they have no competing interests.

\section{References}

1. Chen GC, Yu T, Min XH, Zhao LN, Qing Q, Yuan YH, Su H, Zhan J, Huang KH and Chen QK: Prognosis of 153 patients with decompensated hepatitis B virus-related cirrhosis is improved after 3-year continuous lamivudine treatment. Chin Med J (Engl) 126: 1538-1543, 2013.

2. Lv GC, Yao JM, Yang YD, Zheng L, Sheng JF, Chen Y and Li LJ: Efficacy of combined therapy in patients with hepatitis B virus-related decompensated cirrhosis. World J Gastroenterol 19: 3481-3486, 2013

3. Zhou YM,Zhang XF, Li B, Sui CJ and Yang JM: Prognosis after resection of hepatitis $\mathrm{B}$ virus-related hepatocellular carcinoma originating from non-cirrhotic liver. Ann Surg Oncol 21 2406-2412, 2014.

4. Ming D, Yu X, Guo R, Deng Y, Li J, Lin C, Su M, Lin Z and Su Z: Elevated TGF- $\beta 1 /$ IL-31 pathway is associated with the disease severity of hepatitis B virus-related liver cirrhosis. Viral Immunol 28: 209-216, 2015.
5. Sun LL, Li YH, Chen F, Wang S and Shi H: Study on correlation between liver ultrasonic appearance of patients with chronic hepatitis B and cirrhosis and hydrothorax. Eur Rev Med Pharmacol Sci 20: 5032, 2016.

6. Srivastava M, Rungta S, Dixit VK, Shukla SK, Singh TB and Jain AK: Predictors of survival in hepatitis B virus related decompensated cirrhosis on tenofovir therapy: An Indian perspective. Antiviral Res 100: 300-305, 2013.

7. Wang N, Cao Y, Song W, He K, Li T, Wang J, Xu B, Si HY, Hu CJ and Li AL: Serum peptide pattern that differentially diagnoses hepatitis B virus-related hepatocellular carcinoma from liver cirrhosis. J Gastroenterol Hepatol 29: 1544-1550, 2014.

8. Öcal S, Korkmaz M, Harmanc1 Ö, Ensaroğlu F, Akdur A, Selçuk H, Moray G and Haberal M: Hepatitis B- and hepatitis D-virus-related liver transplant: Single-center data. Exp Clin Transplant 13 (Suppl 1): 133-138, 2015.

9. Niro GA, Ippolito AM, Fontana R, Valvano MR, Gioffreda D, Iacobellis A, Merla A, Durazzo M, Lotti G, Di Mauro L, et al: Long-term outcome of hepatitis B virus-related chronic hepatitis under protracted nucleos $(\mathrm{t})$ ide analogues. J Viral Hepat 20: 502-509, 2013.

10. Wu ZW, Lu HF, Wu J, Zuo J, Chen P, Sheng JF, Zheng SS and Li LJ: Assessment of the fecal lactobacilli population in patients with hepatitis B virus-related decompensated cirrhosis and hepatitis B cirrhosis treated with liver transplant. Microb Ecol 63: 929-937, 2012.

11. Kim IS, Mun JI,KooJH,Kang CJ,BakJK, Cheong JY and Cho SW: Entecavir therapy for patients with hepatitis B virus-related decompensated cirrhosis. Korean J Gastroenterol 59: 224-231, 2012.

12. Zhou J, Yu L, Gao X, Hu J, Wang J, Dai Z, Wang JF, Zhang Z, Lu S, Huang $\mathrm{X}$, et al: Plasma microRNA panel to diagnose hepatitis B virus-related hepatocellular carcinoma. J Clin Oncol 29: 4781-4788, 2011.

13. Sun Y, Lu Y, Xie L, Deng Y, Li S and Qin X: Interferon gamma polymorphisms and hepatitis B virus-related liver cirrhosis risk in a Chinese population. Cancer Cell Int 15: 35, 2015.

14. Saichan X, Wei C, Qinglong F, Jun W and Lei X: Plasma cortisol as a noninvasive biomarker to assess severity and prognosis of patients with craniocerebral injury. Eur Rev Med Pharmacol Sci 20: 3835-3838, 2016.

15. Karra VK, Gumma PK, Chowdhury SJ, Ruttala R, Polipalli SK, Chakravarti A and Kar P: IL-18 polymorphisms in hepatitis B virus related liver disease. Cytokine 73: 277-282, 2015.

16. Bae JS, Kim JH, Pasaje CF, Cheong HS, Lee TH, Koh IS, Lee HS, Kim YJ and Shin HD: Association study of genetic variations in microRNAs with the risk of hepatitis B-related liver diseases. Dig Liver Dis 44: 849-854, 2012.

17. Chu CM and Liaw YF: Hepatitis B virus-related cirrhosis: Natural history and treatment. Semin Liver Dis 26: 142-152, 2006.

18. Hamrahian AH, Oseni TS and Arafah BM: Measurements ofserum free cortisol in critically ill patients. N EnglJ Med 350:1629-1638, 2004.

19. Bárcena R, Domínguez-Antonaya M, López-Sanromán A Martínez-Turnes A, Urman J, del Campo S and Moreno N: Lamivudine therapy of hepatitis B virus-related liver disease: Cirrhosis, post-transplantation recurrence, and de novo infection. Transplant Proc 31: 2457-2458, 1999.

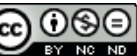

This work is licensed under a Creative Commons Attribution-NonCommercial-NoDerivatives 4.0 International (CC BY-NC-ND 4.0) License. 